\title{
Aumento da obesidade em crianças e adolescentes: risco de complicações cardíacas futuras
}

\author{
Increased obesity in children and adolescentes: risks of future cardiac complications \\ Aumento de la obesidade en niños y adolescentes: riesgo de futuras complicaciones \\ cardíacas
}

\begin{abstract}
Mateus Camargos Silva Alves Simão ${ }^{1 *}$, Abner Fernandes da Silva ${ }^{1}$, André Augusto Dias Silveira ${ }^{2}$, Isabela de Angelles Floro Alonso ${ }^{3}$, Luiza Medeiros de Godoy ${ }^{4}$, Micael Batista Ribeiro Santos ${ }^{4}$, Paloma Barros Aquino Silva ${ }^{3}$, Roberto Homen Adjuto Faria ${ }^{4}$, Vitória Coutinho dos Santos ${ }^{3}$, Adriel Gustavo Lopes ${ }^{1}$.
\end{abstract}

\section{RESUMO}

Objetivo: Discutir sobre o aumento da obesidade infantil e adolescente, correlacionando aos riscos cardiovasculares que essa doença pode causar no adulto. Revisão Bibliográfica: A obesidade infantil cresce, no mundo, de forma alarmante. No Brasil é considerado um dos principais problemas de saúde pública, pelo fato dessa patologia trazer sérios riscos na vida infanto-juvenil e principalmente na vida adulta, dentre eles: diabetes, hipertensão, doença arterial coronariana (DAC), acidente vascular cerebral (AVC), entre outros. Além disso, deve-se ressaltar que mais da metade das crianças obesas se tornam adultos também obesos, e com isso aumenta a quantidade de cardiopatas. Considerações Finais: Visto que cardiopatias são a principal causa de morte mundial e que a obesidade infantil se relaciona diretamente com essas patologias no adulto, faz-se necessário a realização de estudos longitudinais para acompanhar a saúde dessas crianças de forma contínua durante a vida. A partir disso, será possível implementar melhores medidas preventivas com objetivo de diminuir a incidência de doenças cardíacas na vida adulta.

Palavras-chave: Obesidade infantil, Adolescente, Riscos cardiovasculares, Cardiopatias.

\begin{abstract}
Objective: to discuss the increase of childhood and adolescent obesity associated with cardiovascular risks that this disease can cause in adults. Literature Review: Childhood obesity is growing at an alarming rate throughout the world. In Brazil, it is already considered one of the main public health problems as this pathology causes several risks to all age groups, including children, adolescents and especially adults. Among these risks: diabetes, hypertension, coronary artery disease (CAD), stroke are just a few examples. In addition, it should be noted that more than half of the obese children become obese adults as well, thereby increasing the number of cardiac patients. Final Considerations: Since heart diseases are the main cause of deaths in the world and childhood obesity is directly related to this pathology in adults, it is necessary to conduct longitudinal studies, to monitor the health situation of these children continuously through their lives. Thus, it will be possible to implement better preventive measures, in order to reduce the incidence of heart disease during adulthood.
\end{abstract}

Keywords: Childhood obesity, Teenager, Cardiovascular risks, Heart diseases.

\footnotetext{
1 Pontifícia Universidade Católica de Minas Gerais (PUC-MG), Betim - MG.

*E-mail: camargosmateus@gmail.com

2 Universidade Estadual de Montes Claros (UNIMONTES), Montes Claros - MG.

3 Universidade Federal do Maranhão (UFMA), São Luís - MA.

${ }^{4}$ Centro Universitário Atenas (UniAtenas), Paracatu - MG.
} 


\section{RESUMEN}

Objetivo: Discutir el aumento de la obesidad infantil y adolescente, correlacionándolo con los riesgos cardiovasculares que esta enfermedad puede causar en adultos. Revisión Bibliográfica: La obesidad infantil crece en el mundo de manera alarmante. En Brasil ella es considerada uno de los principales problemas de salud pública, ya que esta patología trae riesgos graves en la fase infanto-juvenil, y especialmente en la edad adulta, entre ellos: diabetes, hipertensión, enfermedad de las arterias coronarias (CAD), accidente cerebrovascular, y otros. Además, cabe destacar que más de la mitad de los niños obesos se convierten en adultos igualmente obesos, y así crece el número de pacientes con problemas cardíacos. Consideraciones finales: Dado que las enfermedades cardíacas son la principal causa de muerte en todo el mundo y que la obesidad infantil está directamente relacionada con estas patologías en adultos, es necesario realizar estudios longitudinales para controlar la salud de estos niños de manera continua a por toda la vida. A partir de esto, será posible implementar medidas preventivas mejores, con el objetivo de reducir la incidencia de enfermedades cardíacas en la edad adulta.

Palabras clave: Obesidad infantil, Adolescente, Riesgos cardiovasculares, Cardiopatía.

\section{INTRODUÇÃO}

Nas últimas décadas, o sobrepeso e a obesidade infantil emergiram como um dos problemas de saúde mais graves tanto de países desenvolvidos como aqueles em desenvolvimento. A obesidade se trata de uma doença crônica, complexa, caracterizada pelo acúmulo de tecido gorduroso localizado ou generalizado (WANDERLEY EN e FERREIRA VA, 2010).

Enquanto o sobrepeso caracteriza-se pelo aumento do peso corporal. Ambos podem ser estimados por diferentes métodos ou técnicas, sendo o Índice de Massa Corporal (IMC) (definido pelo peso em kg dividido pela altura em metros quadrados) mais utilizado e aceito para estudos epidemiológicos, obtido com baixo custo e fácil acesso.

De acordo com o Ministério da Saúde (através da Pesquisa de Vigilância de Fatores de Risco e Proteção para Doenças crônicas por Inquérito Telefônico Vigitel), o sobrepeso se tornou prevalente entre os adultos, representando 55,7\% dessa população, e 19,8\% dessa parcela são obesos (MINISTÉRIO DA SAÚDE, 2019). A porcentagem é acentuada com a idade (maior entre 45 a 54 anos) e com o sexo ( $70 \%$ da população obesa são mulheres). Esse índice, ainda que se mostre distribuído por todas as regiões do país, é proporcionalmente aumentado nos grupos de baixa renda. (PINHEIRO ARO, et al., 2004).

O caráter epidêmico e a prevalência crescente da obesidade ocorrem, na grande maioria dos casos, pela associação de fatores genéticos, ambientais, econômicos e comportamentais. A herança genética parece ser de natureza poligênica, desempenhando papel importante na determinação da suscetibilidade do indivíduo para o ganho de peso, ou seja, as características fenotípicas do indivíduo obeso são resultantes da interação de vários genes, porém essa doença é resultado do estilo de vida, tais como hábitos alimentares inadequados, caracterizados pelo aumento exagerado do consumo de alimentos ricos em gordura e com alto valor energético e sedentarismo, gerado pela redução da prática de atividade física e a introdução de hábitos que não geram gasto calórico, como assistir à televisão, usar videogames e computador, que geralmente levam a um balanço energético positivo, favorecendo o surgimento da obesidade na determinação da obesidade (SBP, 2012).

A maior incidência de obesidade traz diversas implicações no aumento de doenças crônicas do tipo não transmissíveis, como as doenças cardiovasculares (DCV). Nesse quesito é importante destacar que as doenças cardiovasculares são patologias que têm o longo período de progressão, porém o aparecimento dos fatores de risco (alterações no metabolismo lipídico, hipertensão arterial, resistência insulínica, tabagismo, inatividade física e obesidade) é precoce, podendo surgir desde a primeira infância (COSTA IFAF, et al., 2017). 
Tendo isso em vista, durante a fase infanto-juvenil, a presença de dois ou mais dos fatores de risco é o suficiente para o desenvolvimento de um problema cardiovascular nos próximos 10 anos de vida. Isso se dá, porque tais condições, quando associadas, aumentam a extensão e a gravidade de anomalias vasculares, prevalecendo na fase adulta (GASTALDELLI U e BASTA L, 2010).

Nota-se que a obesidade aumenta o risco de doenças como diabetes, problemas cardíacos e vários tipos de câncer. Esses males estão relacionados com as principais causas de morte no Brasil. Estima-se que, com alimentação saudável, prática regular de atividade física e manutenção de peso corporal adequado, aproximadamente $33 \%$ dos casos mais comuns de câncer no Brasil possam ser prevenidos (MARTINS APB, 2018).

Para controle da endemia, existem diversas políticas de saúde preventivas, como a Lei no 13.666, de 16 de maio de 2018, que propõe acrescentar o tema transversal da educação alimentar e nutricional no currículo escolar, visando instruir e restringir a alimentação das crianças e melhorar o estilo de vida (BRASIL, 2018). Entretanto, torna-se necessário refletir se a avaliação exata dos parâmetros de composição corporal é necessária desde o início da idade, para classificar corretamente as anormalidades metabólicas e diminuir a chance de novas comorbidades posteriores. Assim, faz-se necessário o estudo desses riscos para maior direcionamento do tratamento de crianças e adolescentes obesos visando diminuir suas complicações cardíacas futuras.

Sabe-se que a obesidade está relacionada com ações individuais como prática de exercícios regulares e alimentação saudável, sendo assim um problema de saúde pública. O número de hospitalizações e mortes por consequência do sobrepeso e obesidade tem aumentado nos últimos anos, principalmente por causas cardiovasculares. Por isso, é importante estudar seus impactos na saúde e direcionar políticas para sua prevenção.

Tendo em vista este cenário, o artigo tem como objetivo discutir sobre o aumento da obesidade infantil e adolescente, correlacionando aos riscos cardiovasculares que essa doença pode causar no adulto.

\section{REVISÃO BIBLIOGRÁFICA}

\section{Aumento de Obesidade Infantil}

A obesidade infantil é considerada um dos principais problemas de saúde pública brasileira, que vêm crescendo de forma alarmante, com alta prevalência. A doença apresenta diversas etiologias, como genéticas, emocionais, socioeconômicas e culturais, que devem ser consideradas de forma singular, principalmente no Brasil, devido a sua população heterogênea (ROCHA LM, 2013).

Conforme evidenciado pela Pesquisa de Vigilância de Fatores de Risco e Proteção para Doenças crônicas por Inquérito Telefônico (Vigitel), em 2018, do Ministério da Saúde, ocorreu incremento de 67,8\% no número de pessoas obesas no País, de 11,8\% no ano de 2006 para 19,8\% em 2018. Outro aspecto a se notar é que, mesmo o excesso de peso sendo mais prevalente em homens, em 2018, a população feminina apresentou obesidade significativamente maior em relação aos homens, 20,7\% contra 18,7\%. (MINISTÉRIO DA SAÚDE, 2019). No quesito infanto-juvenil nos últimos 30 anos a taxa de obesidade infantil dobrou e, em alguns casos, triplicou. Segundo dados da Organização Mundial da Saúde (OMS), existem mais de 340 milhões de crianças e adolescentes obesos entre 5 e 19 anos (KLISH WJ, et al., 2020).

A prevalência de sobrepeso entre menores de 5 anos aumentou de 4,8\% para 6,1\% entre 1990 e 2014 no país, passando de 31 milhões para 41 milhões de crianças afetadas durante esse período (WHO, 2016). Segundo dados divulgados em março pela Secretaria de Direitos Humanos da Presidência da República, o percentual de crianças entre 5 e 9 anos de idade com excesso de peso chega a 33,5\%. Já na adolescência, o quantitativo é de 20,5\% (PR/SDH, 2015).

O Diabetes Mellitus, doença caracteriza por aumento da glicemia capilar secundária a estado de redução da secreção pancreática de insulina ou pela resistência periférica, anos atrás era uma doença mais comumente encontrada no adulto, entretanto, nas últimas décadas existe uma crescente na prevalência dessa 
doença em crianças e adolescentes. Nesse sentido, é importante ressaltar que o diabetes tipo 2 tem grande relevância nos novos casos de Diabetes Mellitus, chegando a mais de 30\% dos números de novos casos da doença. Devido a isso pode ser possível uma relação entre o aumento da prevalência da obesidade infantil com o desenvolvimento dessa doença (OLIVEIRA CL, et al., 2004).

Um estudo multicêntrico realizado pela Universidade de Yale, nos Estado Unidos entre 1999 e $2001 \mathrm{com}$ 55 crianças e 112 adolescentes, sendo todos eles obesos, foi possível constatar a diminuição da tolerância a glicose em $25 \%$ nas crianças e $21 \%$ nos adolescentes. Além disso $4 \%$ dos desse último grupo já eram diabéticos tipo 2 (OLIVEIRA CL, et al., 2004).

A resistência à insulina em conjunto com a hirperinsulinemia são fatores de risco significativos para a diminuição da tolerância à glicose para obesidade pueril. Isso se confirma em razão da resistência à insulina ser um forte prenunciador da diminuição da tolerância à glicose. Aliás, o decurso do desenvolvimento da Diabetes Mellitus na infância parece progredir mais rapidamente quando comparado com a doença no adulto (MILECH A, et al., 2016).

Fato importante é que a fim de evidenciar a obesidade e o excesso de peso na população em geral, utilizase o IMC. Desta forma, através dele pode-se estratificar os pacientes de acordo com sua relação peso/altura ${ }^{2}$, assim como correlacionar as complicações metabólicas e prever a possibilidade de demais condições de risco a saúde (MINISTÉRIO DA SAÚDE, 2019).

Quanto ao diagnóstico da obesidade, em crianças e adolescentes a classificação de sobrepeso e obesidade a partir do IMC é mais arbitrária, não se correlacionando com morbidade e mortalidade, da forma em que se define obesidade em adultos, porém, está significantemente associado à adiposidade. Devido à variação da corpulência durante o crescimento, a interpretação difere de acordo com sexo e faixa etária (SBEM, 2005). O referencial para classificar o estado nutricional de crianças menores de 5 anos são as curvas de crescimento infantil propostas pela OMS em 2006 e para as crianças de 5 a 10 anos incompletos a referência da OMS lançada em 2007 (SPERANDIO N, et al., 2011).

O índice antropométrico mais recomendado pelo Sistema de Vigilância Alimentar e Nutricional (SISVAN) para avaliação do excesso de peso é o IMC-para-idade, porque a associação para determinar o risco à saúde é mais sensível quando avaliada a relação entre o peso e o quadrado da medida de altura (IMC) do que com a medida isolada da altura (Peso para estatura). Acima do percentil 97 (Escore-z +2 ), a criança apresenta obesidade (BRASIL, 2014).

Outras formas também podem ser utilizadas para estimar a quantidade corporal de gordura, algumas com melhor aplicabilidade clínica do que outras. A medida regional de gordura subcutânea é de fácil realização e pode ser utilizada dentro do próprio consultório. A circunferência abdominal, através de medição com fita métrica em regiões definidas (acima da cicatriz umbilical), serve como outro parâmetro auxiliar na estimativa de gordura corporal, além de ser útil para definição de síndrome metabólica (AYER J, et al.,2015).

A bioimpedância utiliza uma corrente elétrica de baixa intensidade, que diferencia tecidos com maior quantidade de água (menor percentual de adipócitos) de outros com menor percentual de água, utilizando o grau de resistência à passagem desta corrente. Alguns métodos auxiliam em casos específicos e possuem maior valor teórico do que prático. Um exemplo é o uso de raios- $X$ de baixa energia, esta técnica é baseada pelo princípio da absorção diferencial dos raios pelos tecidos, assim distinguindo a massa de tecido adiposo dos demais tecidos (AYER J, et al.,2015).

\section{Dislipidemia como fator de incidência na obesidade}

A obesidade está entre os fatores de associação mais prevalentes da dislipidemia na população infantojuvenil. Assim, considerando que o principal mecanismo patogênico da dislipidemia é a aterosclerose, processo que começa na infância e progride de forma gradual até a vida adulta, a análise do perfil lipídico na população infantil e juvenil permite identificar e intervir prematuramente no risco de desenvolver DCV na vida adulta (SAPUNAR J, et al., 2018). Nesse sentido, destaca-se que aspectos comportamentais de hábitos alimentares e sedentarismo influenciam como fatores determinantes do risco cardiovascular em crianças e adolescentes, como redução do gasto calórico e da prática de atividade física (RÉUTER CP, et al., 2018). 
Foi feito um estudo no sul do Brasil, do tipo transversal, envolveu a participação de 1.254 crianças e adolescentes (7-17 anos), 684 meninas e 570 meninos. Após uma coleta de sangue em jejum prévio de 12 horas, foram coletadas amostras de triglicerídeos (TG), colesterol total (CT) e lipoproteínas (HDL-C). Considerou-se a presença de pelo menos um componente do perfil lipídico alterado para classificar como dislipidemia.

Os resultados do presente estudo mostraram uma associação entre dislipidemia de forma significativa com obesidade e sexo feminino e a presença de sobrepeso/obesidade está associada com elevação de CT e TG, fato preocupante, já que há forte relação da dislipidemia com fatores de risco para eventos cardiovasculares, visto que o LDL é a partícula considerada mais aterogênica, levando à formação de placas de ateroma nas artérias coronarianas. Esse processo começa na infância e pode demorar a se manifestar clinicamente, mas que de certa forma aumenta a chance dessa criança vir a ter eventos cardiovasculares futuramente como, infarto agudo do miocárdio em decorrência desse processo (RÉUTER CP, et al., 2018).

\section{Mortalidade Por Cardiopatias}

A partir de uma pesquisa no ano 2015 , foi estimado que 17,7 milhões de pessoas morreram em decorrência de doenças cardiovasculares (DCV), o que seria $31 \%$ de todas as mortes do mundo. Com base nesses e outros dados, as doenças cardiovasculares são consideradas a principal causa de morte mundial. Entre as 17 milhões de mortes prematuras (que seriam as mortes em indivíduos com menos de 70 anos) por doenças crônicas não transmissíveis, $82 \%$ advém de países com baixa e média renda monetária e $37 \%$ decorrem de doenças cardiovasculares (OPAS, 2017). O tabagismo, dietas não saudáveis e obesidade, sedentarismo e o uso nocivo do álcool são fatores comportamentais de risco para a maioria das DCV, por isso elas podem ser prevenidas por meio de estratégias para o enfrentamento do problema, abordando esses fatores de risco, no que tangencia à dimensão assistencial e de promoção da alimentação saudável, seguindo as principais tendências internacionais (IDEC, 2017).

Crianças obesas são mais propensas ao desenvolvimento de aterosclerose subclínica, intolerância à glicose e pré-diabetes que podem se estender na idade adulta. Além dos fatores de risco para o estilo de vida, como sedentarismo e alimentação não saudável, a obesidade infantil pode levar a taxas igualmente alarmantes de anormalidades cardiovasculares entre os jovens. A obesidade abdominal está associada a fatores de risco cardiovasculares e distúrbios metabólicos como Diabetes Mellitus, dislipidemia e hipertensão arterial sistêmica podendo estar presentes na infância (ALISSA EM, et al., 2020).

\section{Cardiopatias Como Fator Secundário na Infância}

O risco, que é aumentado, da criança e do adolescente permanecer no estado de obesidade quando adultos é uma das principais consequências. Assim, estudos longitudinais relacionam como diretamente proporcionais a duração da obesidade e a morbimortalidade por doenças cardiovasculares (FIGUEIREDO EA, et al., 2019). A doença cardiovascular é a principal causa de mortalidade a nível mundial, representando 17,5 milhões de mortes em 2005, prevendo-se que aumente para 23,6 milhões de mortes até 2030 (FRIEDMANN C, et al., 2012).

Os riscos à saúde relacionados à obesidade normalmente aumentam dentro de cada uma das categorias de IMC; mas, dentro de cada categoria, os riscos à saúde são mais altos em crianças com maior circunferência da cintura (CC) em comparação com aqueles com menor CC. Estima-se que o IMC elevado é associado a $30 \%$ das doenças cardíacas coronarianas (DCC) e acidente vascular cerebral (AVC) e 60\% da doença hipertensiva arterial nos países desenvolvidos (SOMMER A e TWIG G, 2018).

Diante desse panorama, se viu necessário criar escores de estratificação de risco cardiovascular (RCV) capaz de prognosticar eventos cardíacos patológicos. O escore Pathobiological Determinants of Atherosclerosis in Youth (PDAY) estratifica, em indivíduos de 15 a 34 anos, o risco para doenças aterosclerótica precocemente, estabelecendo como premissa que fatores de risco para DCV estão relacionados tanto com as fases iniciais quanto com as fases avançadas das lesões ateroscleróticas na carótida e na aorta abdominal durante a adolescência e início da vida adulta. Mostrando influência sistêmica mesmo anos antes do acometimento cardíaco (COSTA IFAF, et al., 2017). 
A estratificação de risco é alcançada pela soma de valores ordenados a fatores modificáveis e não modificáveis. Os fatores não modificáveis são: sexo e idade. Já os fatores modificáveis incluem: Hemoglobina glicosilada HBA1c, índice de massa corporal (IMC), glicemia jejum (GJ), pressão arterial (PA), tabagismo, colesterol HDL e colesterol não HDL. Se o resultado for superior a 0 ele deve ser inserido no gráfico da probabilidade estimada para lesões graves de órgão alvo da pesquisa - aterosclerose de carótida e na aorta abdominal (COSTA IFAF, et al., 2017).

O escore PDAY é normatizado de maneira exponencial, ao ponto que cada unidade somada altera exponencialmente a chance de lesões. Outro ponto que deve ser abordado é sobre a idade. A cada 5 anos de vida são adicionados no gráfico de forma exponencial 5 pontos. Ao passo que, junto com os fatores modificáveis são equivalentes a 11 anos (COSTA IFAF, et al., 2017).

\section{Alterações nas estruturas Cardiovasculares}

A obesidade infantil está associada a alterações nas estruturas cardiovasculares. Estudos ecocardiográficos em crianças obesas mostraram remodelamento cardíaco com aumento de volume atrial esquerdo, hipertrofia do ventrículo esquerdo (HVE) e aumento do volume diastólico final do ventrículo esquerdo, acompanhados de hipertensão arterial ou não (KLISH WJ, et al., 2020). HVE está associada a IMC >95\% para idade e gênero. A documentação de HVE tem sido usada para identificar crianças com risco de complicações na vida adulta, pois predispõe a eventos cardíacos (SANCHES AA, et al., 2015).

Os dados de imagem de deformação miocárdica sugerem que os valores da tensão do miocárdio são levemente reduzidos em crianças com obesidade. Não se pode afirmar que essas leves alterações na estrutura do miocárdio são preditivas de desfecho cardiovascular adverso na vida adulta, uma vez que não foram publicados estudos de acompanhamento longitudinal a longo prazo sobre (KOOPMAN LP e MERTENS LL, 2014).

Um outro estudo mostrou aumento de mortes por doença coronariana em homens que foram obesos durante a adolescência. Autópsias mostraram que estrias gordurosas arteriais (que precedem lesões ateroscleróticas evidentes) já são identificáveis em crianças obesas (KOOPMAN LP e MERTENS LL, 2014).

Pressão arterial sistêmica elevada está relacionada ao aumento da rigidez arterial e da diminuição da vasodilatação mediada pelo fluxo em crianças. $O$ aumento da pós carga devido, ao aumento da rigidez arterial e/ou aumento da pressão arterial sistêmica, pode provocar o aumento de estresse na parede de Ventrículo Esquerdo e Hipertrofia de Ventrículo Esquerdo. Além disso, a pressão arterial sistêmica elevada também está relacionada ao aumento da espessura da camada íntima média da carótida (KOOPMAN LP e MERTENS LL, 2014).

A hipertrofia do ventrículo esquerdo pode explicar anormalidades precoces de relaxamento e possivelmente contribuir para alterações na complacência miocárdica. Isso foi demonstrado em adultos, pois a hipertensão arterial e o aumento da rigidez vascular contribuem para a disfunção diastólica e diminuem o esforço longitudinal. Essas relações entre obesidade, alterações vasculares e alterações do miocárdio são menos óbvias na população pediátrica (HANEVOLD C, et al., 2004).

Existe uma associação entre duração da obesidade e a redução de danos no coração, sugerindo que se uma criança se tornar obesa em uma idade menor, o efeito na função sistólica do miocárdio é mais significativo (FRIEDMANN C, et al., 2012).

Percebe-se que a obesidade e o excesso de peso causam mudanças importantes na estrutura e no tamanho do coração, comprometendo desta forma o seu funcionamento. A mudança mais significativa encontrada nos estudos foi a hipertrofia do ventrículo esquerdo, pois quanto maior o sobrepeso, maior é o esforço do coração para conseguir bombear o sangue. O sobrepeso é associado ao acúmulo de sódio no organismo, isso contribui para o acúmulo de líquidos e dificulta a circulação. Assim, aumenta o volume sanguíneo circulante e provoca dilatação das veias aumentando a pressão venosa e arterial.

A obesidade está diretamente relacionada com dislipidemias que são as principais causas para a formação de placas de gordura nos vasos sanguíneos. Devido a isso, a trombose é uma complicação comum entre os obesos que dá origem a doenças ligadas ao sistema vascular. 
Sendo assim, torna-se imprescindível pesquisar por doenças metabólicas em crianças obesas, por meio de avaliação antropométrica e IMC, a fim de que se possa combater quaisquer enfermidades provenientes da gordura corporal excessiva, prevenindo doenças cardiovasculares no futuro e, por fim, a morte precoce desses pacientes. Dessa forma, esse estudo poderá contribuir para a criação de políticas a níveis municipais, estaduais ou federais que se direcionam a evitar a obesidade e o sobrepeso de crianças e adolescentes, que pode ocasionar o desenvolvimento futuro das DCV quando adultos. Tais políticas podem ser simples como a promoção de campanhas que estimulem a realização de exercícios físicos e de uma dieta balanceada, contendo frutas, legumes e vegetais, ou podem ser mais complexas como a taxação de bebidas açucaradas, para que estimule a ingestão de água e leite em sua substituição. Assim, espera-se mudar a preocupante realidade do futuro de jovens que crescem obesos e com risco de desenvolver doenças ligadas ao coração quando adultos.

\section{CONSIDERAÇÕES FINAIS}

No presente estudo, encontrou-se elevado risco de doenças cardiovasculares em crianças obesas e constatou-se que elas possuem maiores alterações nas funções cardíacas quando comparadas a crianças eutróficas. Diante disso, deve-se atentar quanto às consequências do excesso de peso nessas crianças, já que isso pode influenciar de forma significativa no aumento da incidência de doenças cardiovasculares e da morbimortalidade na vida adulta. Foi visto em alguns estudos relações entre a obesidade infantil com algumas comorbidades, como a aumento da intolerância à glicose, aterosclerose, DCC e AVC, além de alterações em estruturas cardiovasculares. Entretanto, há a necessidade da realização de novos estudos do tipo longitudinal, a fim de melhor elucidar os possíveis efeitos cardiovasculares futuros para crianças obesas, para que assim, medidas de prevenção possam ser implementadas de forma efetiva nessa faixa etária, seja no rastreio precoce de doenças metabólicas pré-existentes, seja na avaliação antropométrica e IMC, ou novos métodos a serem criados.

\section{REFERÊNCIAS}

1. ADELBORG K, et al. Levels of and Changes in Childhood Body Mass Index in Relation to Risk of Atrial Fibrillation and Atrial Flutter in Adulthood. American Journal of Epidemiology, 2019; 188(4): 684-693.

2. ALISSA EM, et al. Physical activity pattern and its relationship with overweight and obesity in saudi children. International Journal of Pediatrics and Adolescent Medicine, 2020.

3. AMERICAN DIABETES ASSOCIATION. 13. Children and Adolescents: Standards of Medical Care in Diabetes-2020. Diabetes Care, 2020; 43(1): 163-182.

4. AYER J, et al. Lifetime risk: childhood obesity and cardiovascular risk. European Heart Journal, 2015; 36(2): 13711376.

5. BRASIL. 2018. In: Altera a Lei no 9.394, de 20 de dezembro de 1996, para incluir o tema transversal da educação alimentar e nutricional no currículo escolar. Brasília, DF: Presidência da República, 2018a. Disponível em: http://www.planalto.gov.br/ccivil_03/_ato2015-2018/2018/lei/L13666.htm. Acesso em: 05 de mai. de 2020.

6. BRASIL, Ministério da Saúde. Secretaria de Atenção à Saúde. Departamento de Atenção Básica. Protocolos do Sistema Alimentar e Nutricional - SISVAN na assistência à saúde. Brasília: Ministério da Saúde, 2014.

7. COSTA IFAF, et al. Adolescentes: comportamento e risco cardiovascular. Jornal Vascular Brasileiro, 2017; 16(3): 205-213.

8. DATASUS. Sistema de Informação de Mortalidade. Disponível em: http://tabnet.datasus.gov.br/cgi/deftohtm.exe?sim/cnv/obt10br.def. Acesso em: 05 de mai. de 2020.

9. FIGUEIREDO EA, et al. Políticas públicas de educação em saúde para a prevenção de comorbidades e doenças cardiovasculares. Revista da Seção Judiciária do Rio de Janeiro, 2019; 23(45):141-160.

10. FONSECA, ACP. Abordagem epidemiológica e molecular da obesidade em uma amostra do Rio de Janeiro. Tese (Doutorado em Biologia Celular e Molecular) - Instituto Oswaldo Cruz. Fundação Oswaldo Cruz, Rio de Janeiro, 2019; $231 \mathrm{p}$.

11. FRIEDMANN C, et al. Cardiovascular disease risk in healthy children and its association with body mass index: systematic review and meta-analysis. The BMJ, 2012; 345: 4759.

12. GASTALDELLI U, BASTA L. Gordura ectópica e doença cardiovascular: o que é o link?. Nutrition Metabolic \& Cardiovascular Diseases, 2010; 20(7): 481-490.

13. HANEVOLD C, et al. The effects of obesity . gender and ethnic group on left ventricular hypertrophy and geometry in hypertensive children: a collaborative study of the Intenational Pediatric Hypertension Association. Pediatrics, 2004; 113(2): 328-333. 
14. IDEC. 2017. In: Alimentação saudável nas escolas: Guia para municípios. Disponível em: https://idec.org.br/ferramenta/alimentacao-saudavel-nas-escolas. Acesso em: 05 de mai. de 2020.

15. KLISH WJ, et al. Overview of the health consequences of obesity in children and adolescentes. UpToDate, 2020.

16. KOOPMAN LP, MERTENS LL. Impact of Childhood Obesity on Cardiac Structure and Function. Current Treatment Options in Cardiovascular Medicine. Current Treatment Options in Cardiovascular Medicine, 2014; 16(11).

17. MARTÍNEZ-GÓMEZ D, et al. Sedentarismo, adiposidad y factores de riesgo cardiovascular en adolescentes: estudio AFINOS. Revista Española de Cardiologia, 2010; 63(3): 277-285.

18. MARTINS APB. É preciso tratar a obesidade como um problema de saúde pública. Revista de Administração de Empresas, 2018; 58(3), 337-341.

19. MILECH A, et al. Diretrizes da Sociedade Brasileira de Diabetes (2015-2016). AC Farmacêutica, 2016.

20. MINISTÉRIO DA SAÚDE. Brasileiros atingem maior índice de obesidade nos últimos treze anos. 2019.

21. MITTELMAN SD, et al. Adiposity predicts carotid intima-media thickness in healthy children and adolescents. Jornal Pediatria, 2010; 156(4): 592-597.

22. OLIVEIRA CL, et al. Obesidade e síndrome metabólica na infância e adolescência. Revista de Nutrição, 2004; 17(2): 237-245.

23. OPAS. 2017. In: Doenças cardiovasculares. OPAS Brasil. Disponível em: https://www.paho.org/bra/index.php?option=com_content HYPERLINK. Acesso em: 05 de mai. de 2020.

24. PARKER ED, et al. Change in Weight Status and Development of Hypertension. Pediatrics, 2016; 137(3).

25. PINHEIRO ARO, et al. Uma abordagem epidemiológica da obesidade. Revista de Nutrição, 2004; $17(4): 523-533$.

26. PRESIDÊNCIA DA REPÚBLICA, SECRETARIA DE DIREITOS HUMANOS. 2015. In: SDH/PR apresenta dados sobre alimentação adequada de crianças e adolescentes no Brasil. Disponível em: https://www.gov.br/mdh/pt$\mathrm{br} / \mathrm{sdh} /$ noticias/2015/marco/copy_of_270215AvisodePautaAlimentaoadequada2.pdf/view. Acesso em: 05 mai. de 2020.

27. RÉUTER CP, et al. Relação entre Dislipidemia, Fatores Culturais e Aptidão Cardiorrespiratória em Escolares. Arquivo Brasileiro de Cardiologia, 2019; 112(6): 729-736.

28. ROCHA LM. Obesidade Infantil: uma revisão bibliográfica. Trabalho de conclusão de Curso- Universidade Federal de Minas Geral, Belo Horizonte, 2013; 49p.

29. SANCHES AA, et al. Markers of cardiovascular risk, insulin resistance, and ventricular dysfunction and remodeling in obese adolescents. Jornal Pediatria, 2015; 166(3): 660-665.

30. SAPUNAR J, et al. Alta prevalencia de dislipidemias y riesgo aterogénico en una población infanto-juvenil. Revista médica de Chile, 2018; 146(10): 1112-1122.

31. SAUNDERS TJ, et al. Sedentary behaviour as an emerging risk factor for cardiometabolic diseases in children and youth. Canadian Journal of Diabetes, 2014 ; 38(1): 53-61.

32. SHAH AS, et al. Clustering of Risk Factors: A Simple Method of Detecting Cardiovascular Disease in Youth. Pediatrics, $2011 ; 127(2): 312-318$.

33. SOCIEDADE BRASILEIRA DE ENDOCRINOLOGIA E METABOLISMO. Obesidade: Diagnóstico e Tratamento da Criança e do Adolescente. Projeto Diretrizes, 2005.

34. SOCIEDADE BRASILEIRA DE PEDIATRIA. Manual de orientação: Departamento de nutrologia: alimentação do lactente ao adolescente, alimentação na escola, alimentação saudável e vínculo mãe-filho, alimentação saudável e prevenção de doenças, segurança alimentar. Rev. amp. Departamento Científico de Nutrologia, 3. ed., Rio de Janeiro, 2012.

35. SOMMER A, TWIG, G. The Impact of Childhood and Adolescent Obesity on Cardiovascular Risk in Adulthood: a Systematic Review. Current Diabetes Reports, 2018; 18(10).

36. SPERANDIO N, et al. Comparação do estado nutricional infantil com utilização de diferentes curvas de crescimento. Revista de Nutrição, 2011; 24(4): 565-574.

37. STEVE D, et al. Adiposity Predicts carotid intima-media Thickness in healthy crildren and adolescents. The journal of pediatrics, 2010; 156(4): 592-597.

38. TIROSH A, et al. Adolescent BMI trajectory and risk of diabetes versus coronary disease. The New England Journal of Medicine, $2011 ; 364: 1315-1325$.

39. WANDERLEY EN, FERREIRA VA. Obesidade: uma perspectiva plural. Revista Ciência \& Saúde Coletiva, 2010; 15(1): 185-194.

40. WORLD HEALTH ORGANIZATION. 2016. In: Report of the commission on ending childhood obesity. WHO Library Cataloguing-in-Publication Data. Disponível em: https://www.who.int/end-childhood-obesity/publications/echoreport/en/. Acesso em: 05 de mai. de 2020. 